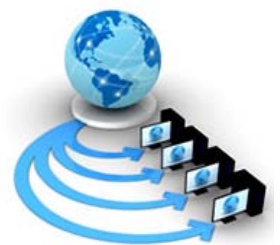

Volume 9, No. 2, March-April 2018

International Journal of Advanced Research in Computer Science

REVIEW ARTICLE

Available Online at www.ijarcs.info

\title{
COOPERATIVE MULTI-AGENT LEARNING IN SOCIAL NETWORKS: A REVIEW
}

\author{
Ginni Devi $\square$ \\ Dept of Comp. Sci. \& Engg., \\ Guru Nanak Dev University Regional Campus, \\ Gurdaspur, Punjab , India
}

\author{
Harjot Kaur \\ Dept of Comp. Sci. \& Engg., \\ Guru Nanak Dev University Regional Campus, \\ Gurdaspur, Punjab, India
}

\begin{abstract}
Coordination in cooperative multi-agent systems is one of the important issues in multi-agent learning and has been broadly studies in the literature. Much work in this field has been carried out yet. However, there are still some coordination issues that are required to be improved. In this work, we look over the multi-agent coordination problems in cooperative environments under the networked multi-agent learning framework using some social network structures and will try to improve coordination efficiency. In our framework, we talk about two types of learners, i.e. individual action learner and joint action learner. We are considering hierarchical multi-agent learning framework to accelerate the coordination efficiency. Our research direction is to consider utilizing the characteristics of different neighboring agents (e.g., the past interaction histories of different nodes, the relative degree of different nodes in the neighborhood) while performing the multi-agent learning to improve coordination performance.
\end{abstract}

Keywords: Multi-agent Systems, Social networks, Multi-agent Learning, Coordination, Neighbouring Agent characteristics.

\section{INTRODUCTION}

According to Michael Wooldridge [17], "An agent is a computer system that is situated in some environment, and that is capable of autonomous action in this environment in order to meet its design objectives".

"Multi-agent systems (MAS) are distributed systems of independent actors, called agents that cooperate or compete to achieve a certain objective. These agents may be computer programs, robots, or even humans" [18]. They can be utilized to take care of issues that are severe or impossible for an individual agent or a monolithic system to solve.

A cooperative multi-agent system (CMAS) consists of a set of autonomous agents who interact with each other in a shared environment. In order to successfully interact, agents in MAS will have the ability to cooperate, coordinate, and negotiate with other resident agents accordingly, in much the same manner we cooperate, coordinate, and negotiate with other people in our daily lives. One important property of an agent in a multi-agent system is its ability of adaptively adjusting its behaviors in response to other agents so as to achieve effective coordination on desirable outcomes since the outcome not only rely on the action it takes but also the action taken by other agents that it interacts with. In cooperative MASs, the agents share common interests with the same reward function, thus the increase in individual's benefit also leads to the increase in the benefits of the whole group. Hao and Leung[6] were the first who proposed a multi-agent social learning framework to investigate multi-agent coordination problem in cooperative games assuming that the agents' interactions are random. In their recent work, they considered the underlying network structure instead of random interaction mechanism to facilitate more efficient coordination.
To this end, in this work, we have studied various previous works that have been done in order to improve the coordination in the cooperative multi-agent system under multi-agent learning frameworks. We have also proposed a networked multi-agent learning framework to investigate the multi-agent coordination problem and improve multi-agent coordination efficiency in cooperative MASs by modeling various network topologies in an explicit manner and considering some characteristics of neighboring agents of a particular agent. In this framework, each agent learns its policy from its neighboring agents via repeated interactions within the system. We shall consider a number of representative social network structures, i.e. ring network, small-world network, and scale-free network. During each round, each agent interacts with another agent randomly selected from the neighborhood, and the interactions between each pair of agents are represented as two-player cooperative Markov games. If no underlying topology exists, then one agent is randomly selected as its partner from the population. Each agent learns its policy simultaneously over repeated interactions with randomly selected agents from its neighborhood. Besides, apart from learning from its own experience, each agent may also learn from the experience of its neighbors.

We believe that more work needs to be done on top of all the previous frameworks to improve the coordination efficiency in different scenarios. Therefore, we are utilizing the characteristics of different neighboring agents (e.g. the relative degree of different nodes in the neighborhood, the past interaction histories of different nodes) when performing the multi-agent learning (the observation mechanism) to improve the coordination performance. This is one of the unique characteristics of network-based multi-agent learning which is 
expected to further improve the coordination of agents on optimal outcomes.

The rest of the paper proceeds as follows: The literature review corresponding to various multi-agent learning approaches and social networks using MAS is presented in Section 2. Section 3 outlines the problem definition, i.e., Cooperative multi-agent Learning in social networks, Section 4 explains the proposed model that comprises of two parts, i.e., multi-agent learning framework and coordination model which is based upon the number of steps that will result in coordination improvement and conclusions are presented in Section 5.

\section{LITERATURE SURVEY}

Claus and Boutilier[1] examined some facts that can affect the dynamics of the multi-agent Q-learning. Two forms of multiagent reinforcement learners, independent learners (ILs) and joint action learners (JALs) were distinguished and compared with each other. They studied (a simple form of reinforcement learning) Q-learning in cooperative multi-agent systems under the two perspectives, the first one focused on the influence of that game structure including exploration strategies on convergence to Nash equilibria. Alternative optimistic exploration techniques were also proposed in this work that increased the probability of convergence to an optimal equilibrium.

Franchi[3] demonstrated a multi-agent system which implemented a fully distributed Social Network System(SNS). This system helped the users to store their profiles as FOAF (Friend Of A Friend) profiles. FOAF was basically an extensive machine-readable ontology that gave a description of the person, their activities, and relation to other persons. The main idea was that "user should be the sole owner of the information they provide to the system" and they could specify which data should be used to construct their social network. In addition, there should not be any centralized database, so system could not edit the profile against the user's will. A connection discovery algorithm has been discussed that used the stored information in the user's profile in order to construct a social network on its owner's behalf. The proposed system also preserved the privacy of the user's information in their profile. Moreover, the author has also proposed the design of an implementation based on the HDS (Heterogeneous Distributed System) framework.

Franchi and Poggi[4] have represented the relationship between multi-agent systems and social networks by introducing various multi-agent system models and techniques and discussed how they have been used in the development of social network systems. The authors have presented the work which applied multi-agent simulation to the social networks and also reviewed the work which considered social networks as a conceptual model in favor of improvement in simulation results. This research raised various improvements in need of simplified development methods of agent-based modeling and their simulation.

Tuyls and Weiss[15] authored the article that was able to answer the question what MAL is really about. They introduced some basics of Multiagent learning and deeply explored the nature of multiagent learning. They discussed some milestones of the field by sketching the main research developments in MAL since the end of the 1980s. Moreover, several recent articles have been considered by the authors who have done an excellent job of surveying and identified the main challenges that MAL field was facing. In order to overcome some of the issues regarding current topics, they investigated a wide range of learning paradigms such as transfer learning and swarm intelligence, that could be extended to further nourish the relationship between singleagent and multi-agent learning research. The findings from this study have made several contributions to the currently existing literature.

Hao and Leung[5] investigated the multi-agent coordination problem by proposing two types of learners (IALs and JALs) in cooperative environments under the social learning framework, which was complementary to the large body of previous work in the framework of repeated interactions among fixed agents. The learning performance of both types learners was evaluated by authors by using a number of rigorous cooperative games, and the resulting influence of the degree of information sharing degree upon the performance of learning and reasoning was analyzed as well.

Hao and Leung[6] proposed a framework that was based upon social learning for a population of agents in order to coordinate on socially optimal outcomes. After surveying a number of papers, the authors found that the previous agent's decision making processes were based on evolutionary learning, which resulted in high communication cost and high deviation on the coordination rate. Considering this drawback, a new mechanism i.e., observation mechanism has been introduced by them into the social learning framework to reduce the amount of communication among agents. In addition to that, they developed agent's learning strategies that were based on reinforcement learning technique instead of evolutionary learning. By implementing this technique, the agents were able to achieve much more stable coordination on socially optimal outcomes as compared to the previous approaches. The performance evaluation of this social learning framework was extensively done under the testbed of two-player general-sum games and was compared with previous work [10][14]. The influences of different factors on the learning performance of the social learning framework were investigated as well.

Hao and Leung[8] had investigated the multi-agent coordination problem in cooperative environments under the social learning frameworks. Two different types of learners (IALs and JALs) based on the traditional Q-learning algorithm were introduced by incorporating both heuristics of optimal assumption and Frequency Maximum Q value(FMQ)[13] strategy. The researchers concluded that for deterministic cooperative games, both IALs and JALs could effectively learn to coordinate with optimal joint actions without significant performance difference, however, when it comes to stochastic cooperative games, JALs usually achieve much better performance than IALs, since it they better distinguish between the stochasticity of the game itself and the stochastic explorations of various interacting agents.

Jiang et al.[11] presented a novel task allocation model based on the negotiation reputation mechanism, where an agent's past behavior in the resource negotiation of task execution 
could influence its probability to allocate new tasks in the future. The researchers have contributed by presenting a systematic research on task allocation in an undependable multi-agent system in a social network(MAS-SN) for the first time with the aim of achieving dependable resource access and minimizing the tasks' necessary resource access time at the same time. They have also presented some task allocation objectives. In order to meet them, a negotiation reputation based allocation mechanism and a reward/punishment mechanism has been designed to ensure that the truthful agents with smaller communication distances have higher probabilities of receiving tasks. In addition to that, load balancing was adopted in task allocation so that the problem of waiting time at heavy-burdened agents could be alleviated. Moreover, through the experiments of their model on typical networks, the authors concluded that their model could perform better on typical social network structures.

Jiang and Jiang[12] answered the problem related to a social network that how to connect social networks and multi-agent systems and how to use multi-agent technologies to model and analyze social networks, by surveying social networks from a multi-agent perspective. The authors mainly reviewed the various related studies to the actor-oriented view and the actorstructure crossing view. Since coordination is critical for both multi-agent systems and social networks, this paper proposed that coordination mechanisms could be used as links between research on multi-agent systems and research on social networks. Therefore, this work mainly categorized various relevant studies on social networks based on the coordination mechanisms among the actors in the social networks, that mainly included three typical categories: cooperative social networks, non-cooperative social networks, and multiple social networks. Then, for each class, review of the existing studies and discussion of their relationship with corresponding multi-agent systems was done. Various challenging issues and also discussed some of the challenging issues and possible future research directions were also cited in this work. This survey established a very close relationship between social networks and multi-agent systems and it could be well understood well via a multi-agent coordination perspective. Moreover, the authors also compared the multiagent perspective with another perspective on studying social networks and explored some pros and cons of the multiagent coordination perspective.

Wang and Jiang[16] contributed towards proposing a distributed social task allocation model for the first time with the aim of both load balancing and maximizing social effectiveness. The given model was capable of being scaled in order to reduce the computation time and was robust in adapting the system dynamics as well. In this work, the authors addressed the complex task allocation in social networks, where a set of individuals should work together to satisfy a complex task's skill requirements. The presented experimental results demonstrated that the proposed model not only produced as less task execution cost as the benchmark centralized models but also reduced the computation time significantly as compared to the traditional models.

Hao et al.[7] studied the multi-agent co-ordination problem in cooperative games under the networked social learning framework. This work was the first initiative towards considering the underlying topology to achieve efficient coordination on optimal outcomes in different cooperative environments. The presented framework basically focused on two representative social network topologies: the small-world network and scale-free network topologies. In this framework, each agent was able to interact with a very small proportion of agents. And also various agents interact with different proportions of agents depending on their own degrees of connection. To the end, the authors finally evaluated the learning performance of both types of learners: IALs and JALs in various types of cooperative games within the learning framework for both topologies.

Choudhury et al.[2] introduced a novel opinion dynamics model that reproduced different behaviors observed in social networks such as dissensus, clustering, oscillations, opinion propagation etc. The work presented two trust models, i.e. continuous opinions with continuous actions(COCA) and continuous opinions with discrete actions(CODA) to handle two distinct situations that were considered in the presented work. In order to more precisely outline the opinion dynamics as well as to recover more sensible behaviors, a mix of continuous opinion with discrete actions (CODA) was also proposed. The CODA model assumed that each individual agent has access only to the actions of its neighbors. But COCA model assumed that each individual agent has access to the opinions of its neighbors. The main outcome of this work provided the features of preservation along with diffusion of actions under general communication topologies. A complete analysis of the opinions' behavior was performed in the particular instances of complete and ring communication graphs.

Hao et al.[9] carried out a systematic investigation about how agents could effectively learn so as to coordinate on an optimal policy in various cooperative multi-agent environments under the networked social learning framework. In their framework, they proposed two types of learners: IALs and JALs. In their previous work, the authors have introduced the networked social learning framework that basically concentrated on only two representative social network topologies: the small-world and scale-free network topologies and resulted in successfully improved the coordination among agents. In this article, they just extended the previous framework by considering two more representative topologies: Random and Ring networks. After a systematic investigation, they concluded that the network topology has a significant impact on the learning performance among agents and the framework introduced by them has literally accelerated the coordination among agents. In general, JALs are able to achieve better coordination performance than IALs. Their framework was basically focused on four representative topologies. The influence of various network topologies and various network topology factors on the learning performance of agents was investigated in this work. The experimental results showed that the underlying topologies were indeed counted in terms of the success rate of coordination and the convergence rate.

We have reviewed set of previous papers which reported recent applications of agents in the field of social learning. The previous studies had systematically investigated coordination problems in cooperative environments and various learning strategies had been proposed by them in order to improve 
coordination performance. We have also investigated how coordination had been improved sequentially by using these strategies. In the recent work, to improve coordination, a networked multi-agent learning framework had been introduced, which consists of a number of agents and each agent interacts with another agent selected in a random manner from its neighborhood in each round. Under this framework, two types of learners were proposed IALs and JALs which resulted in improved coordination performance. We will further try to improve coordination by extending the previously modeled framework by introducing two different neighboring agent characteristics into it, i.e. past interaction histories and relative degree of interaction of agents with other agents, which is expected to facilitate and improve the coordination of agents in a cooperative multi-agent system.

\section{PROBLEM DEFINITION: COOPERATIVE MULTI-AGENT LEARNING IN SOCIAL NETWORKS}

We have investigated that an agent has a unique property of adaptiveness, i.e., it can adjust its behaviors according to others behavior in order to achieve effective coordination on desired outcomes. One widely studied class of coordination problem is how to coordinate with cooperative MASs, in which the agents share common interests with the same reward function. Initially, no underlying topology was considered in the social learning framework, which thus could not fully reflect the interaction in practical multi-agent systems. So, to make the coordination techniques applicable in practice especially for those MAS applications closely residing in existing social networks, Hao et al.[9] considered the underlying topologies of interaction environment when designing coordination techniques. Our research direction is to consider utilizing the characteristics of different neighboring agents (e.g., the relative degree of nodes in the neighborhood, the past interaction histories of different nodes) when performing the multi-agent learning to improve coordination performance. This is one of the unique characteristics of network-based multi-agent social learning which is expected to be able to facilitate the coordination of agents on optimal outcomes.

\section{PROPOSED MODEL}

The proposed model for enhancing coordination in the networked multi-agent system comprises of two parts, i.e., Multi-agent Learning Framework and Coordination Model as described below.

\section{A. Multi-agent Learning Framework}

We have proposed a multi-agent learning framework which consists of a population of agents, who interact with each other and randomly selected neighboring agents from neighborhood in a cooperative multi-agent system (Fig. 1). We are using multi-agent reinforcement learning to resolve all the coordination issues. In the networked multi-agent learning, we have considered two types of learners, individual action learners (IALs) and joint action learners (JALs). They will use observation mechanism during the interaction that will allow the agents to observe the characteristics of other agents. The interaction networks are introduced, that can influence the possible interactions among different agents and also the amount of perceived information by each agent in the system.
We are focusing on the following realistic social network topologies for the agent interactions: Random, Ring Network, Small-world Networks (SWN) and Scale-free Networks (SFN). Next, we are considering two characteristics of different agents which exist in the neighborhood of a particular agent, i.e., past interaction histories and relative degree of nodes in the neighborhood. Then, a coordination model is introduced by us that will result in improved coordination of agents in networked multi-agent learning.

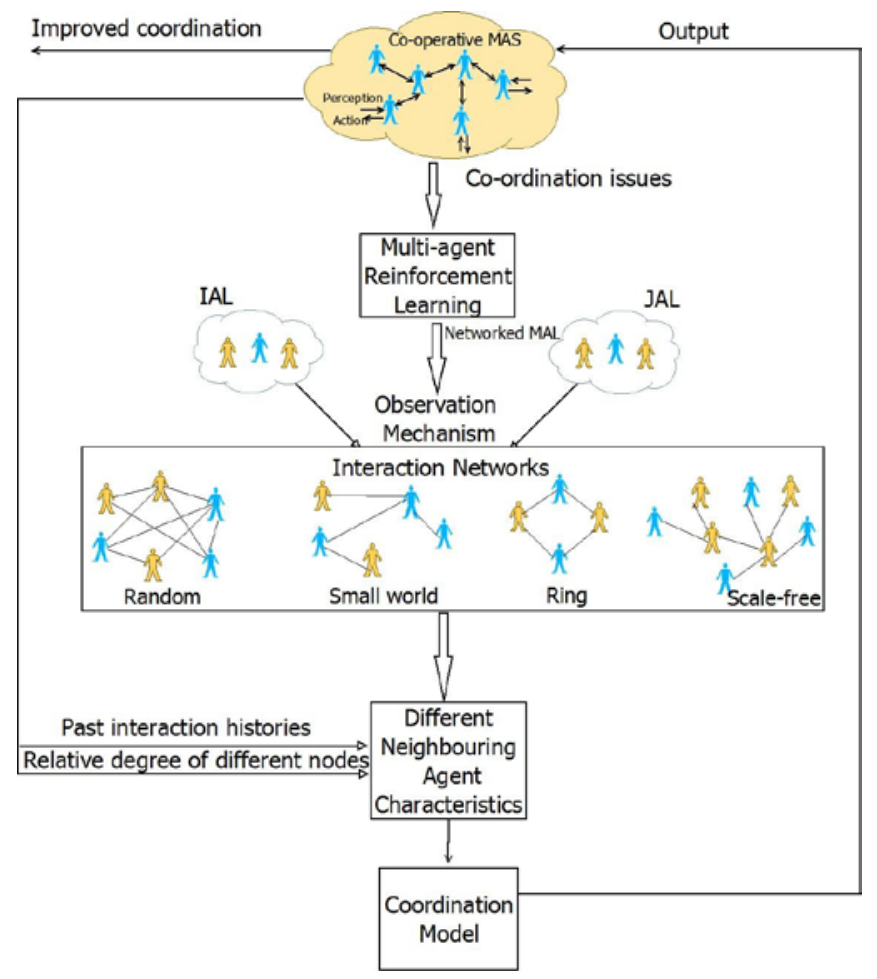

Fig 1: Multi-agent Learning Framework

\section{B. Coordination Model}

Secondly, we have proposed a coordination model (Fig. 2), which consists of a number of agents with common goals who will participate in the network. The agents will perform tasks for which they receive units of rewards. All the agents who will participate in interaction will come under the common action space that consists of a number of cooperative tasks with different policies. A decision-making model will be used to choose the best joint optimal action/policy by comparing all the available actions corresponding to a required reward. The next step will be to learn policies by observation and interactions in order to achieve better coordination. During performance evaluation, coordination problems will be checked after learning by using test beds, and we will check whether the performance gets improved or not. Furthermore, the updation of learning policies after the interaction of each agent with its neighbors will be done in order to improve coordination. In this way, we can improve the coordination by following various steps with repeated interactions. 


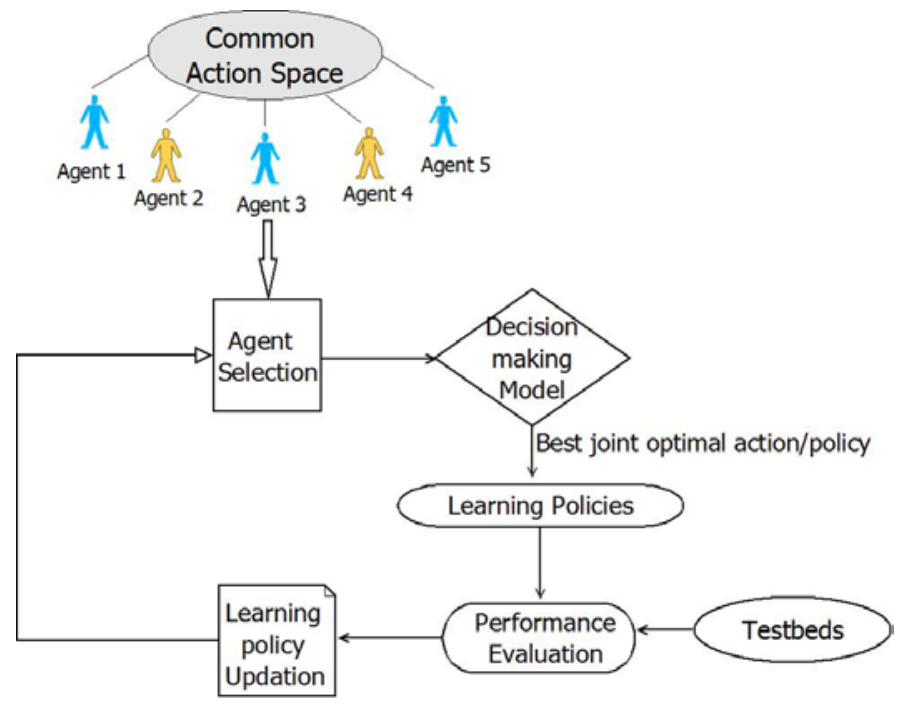

Fig 2: Coordination Model

\section{CONCLUSION}

In this work, we have reviewed a set of several research papers mainly published in indexed journals and conferences, which have reported recent applications of agents in the social learning domain. The current studies of multi-agent learning have been reviewed by us considering step by step improvement including various techniques in order to improve coordination. It is systematically investigated that how coordination efficiency can be improved while performing the network-based multi-agent learning. The findings that we have presented suggest that by utilizing the characteristics of different neighboring agents when performing the multi-agent learning, the coordination performance can be improved. Therefore, on the basis of this idea, we have proposed a multiagent learning framework. Our framework can be considered as the first and fundamental step to improve coordination in networked multi-agent learning using the characteristics of neighboring agents. This is a novel approach followed in network-based multi-agent learning which is expected to facilitate the coordination of agents on optimal outcomes.

\section{REFERENCES}

[1] Claus, C., and Boutilier, C. 1998. The dynamics of reinforcement learning in cooperative multiagent systems. In Proceedings of the Fifteenth National Conference on ArtificialIntelligence, 746-752.

[2] Chowdhury, N. R., Morarescu, I., Martin, S., \& Srikant, S. (2016). Continuous opinions and discrete actions in social networks: A multi-agent system approach. 2016 IEEE 55th Conference on Decision and Control (CDC).
[3] Franchi, E. (2010) A Multi-Agent Implementation of Social Networks. Proceedings of WOA 2010 Undicesimo Workshop Nazionale “Dagli Oggetti agli Agenti”, Rimini.

[4] Franchi, E., \& Poggi, A. (n.d.). Multi-Agent Systems and Social Networks. Handbook of Research on Business Social Networking, 84-97. doi:10.4018/978-1-61350-1689.ch005

[5] Hao, J., \& Leung, H. (2013) The dynamics of reinforcement social learning in cooperative multiagent systems. In Proceedings of IJCAI 13, Beijing, pp 184-190

[6] Hao, J., \& Leung, H. (2013). Achieving Socially Optimal Outcomes in Multiagent Systems with Reinforcement Social Learning. ACM Transactions on Autonomous and Adaptive Systems,8(3), 1-23. s

[7] Hao, J., Huang, D., Cai, Y., \& Leung, H. (2014). Networked Reinforcement Social Learning towards Coordination in Cooperative Multiagent Systems. 2014 IEEE 26th International Conference on Tools with Artificial Intelligence. doi:10.1109/ictai.2014.63

[8] Hao, J., \& Leung, H. (2013) Reinforcement Social Learning of Coordination in Cooperative Multiagent Systems(extended abstract). In: Proceedings of AAMAS' 13, Saint Paul, pp 1321-1322.

[9] Hao, J., Huang, D., Cai, Y., \& Leung, H. (2017). The dynamics of reinforcement social learning in networked cooperative multiagent systems. 2014 IEEE 26th International Conference on Tools with Artificial Intelligence. In: Proceedings of AAAI'58, 2017, pp. 111122.

[10] Hao, J. Y. and Leung, H. F. 2011. Learning to achieve social rationality using tag mechanism in repeated interactions. In Proceedings of ICTAI'11. 148-155.

[11] Jiang, Y., Zhou, Y., \& Wang, W. (2013). Task Allocation for Undependable Multiagent Systems in Social Networks. IEEE Transactions on Parallel and Distributed Systems, 24(8), 1671-1681.

[12] Jiang, Y., \& Jiang, J. C. (2014). Understanding Social Networks From a Multiagent Perspective. IEEE Transactions on Parallel and Distributed Systems, 25(10), 2743-2759.

[13] Kapetanakis, S. and Kudenko, D. 2002. Reinforcement learning of coordination in cooperative multi-agent systems. In Proceedings of the AAAI'02. 326-331.

[14] Matlock, M. and Sen, S. 2007. Effective tag mechanisms for evolving coordination. In Proceedings of AAMAS'07. 1340-1347.

[15] Tuyls, K., \& Weiss, G. (2012). Multiagent Learning: Basics, Challenges, and Prospects. AI Magazine, 33(3), 41.

[16] Wang, W., \& Jiang, Y. (2015). Multiagent-Based Allocation of Complex Tasks in Social Networks. IEEE Transactions on Emerging Topics in Computing, 3(4), 571-584. doi:10.1109/tetc.2015.2403200

[17] Wooldridge, Michael. An introduction to multiagent systems. John Wiley, 2002.

[18] “Multi-Agent system.” Wikipedia, Wikimedia Foundation, 5 Jan. 2018, en.wikipedia.org/wiki/Multiagent_system. 